Rev. Int. Contam. Ambie. 38, 11-26, 2022

https://doi.org/10.20937/RICA.54073

\title{
COMPORTAMIENTOS DE AUTOPROTECCIÓN FRENTE A LA CONTAMINACIÓN DEL AIRE Y FACTORES PSICOSOCIALES, TEMUCO, CHILE
}

Self-protective behaviors against air pollution and psychosocial factors, Temuco, Chile

\author{
Boris ÁLVAREZ ESCOBAR ${ }^{1}$, Pamela CASTILLO FARINA ${ }^{2}$, \\ Javiera NAVARRO-RIFFO ${ }^{2}$, Cindy MUÑOZ MUÑOZ ${ }^{2}$ y Álex BOSO GASPAR ${ }^{1 *}$
}

${ }^{1}$ Núcleo Científico Tecnológico en Ciencias Sociales y Humanidades, Universidad de la Frontera, Av. Francisco Salazar 01145, Temuco, Chile, Código Postal 4811230.

${ }^{2}$ Universidad Católica de Temuco, Manuel Montt 56, Campus San Francisco, Temuco, Chile, Código Postal 4813302.

*Autor para correspondencia: alex.boso@ufrontera.cl

(Recibido: julio de 2020; aceptado: marzo de 2021)

Palabras clave: conductas autoprotectoras, percepción de riesgo, calidad del aire, salud

\section{RESUMEN}

Las ciudades intermedias del sur de Chile sufren cada invierno episodios críticos por la emisión de grandes cantidades de $\mathrm{MP}_{2.5}$ procedentes de la combustión de estufas y cocinas a leña. Los responsables políticos han implementado planes de descontaminación atmosférica, que incluyen campañas de sensibilización para promover conductas autoprotectoras como el uso de mascarillas o realizar ejercicios sólo en intramuros. El objetivo de este estudio fue analizar el rol de los factores psicosociales en la adopción de conductas autoprotectoras frente a la contaminación del aire en residentes de Temuco y Padre Las Casas. Para ello, se llevó a cabo un estudio de diseño no experimental, transversal y de alcance correlacional, a partir de una muestra no aleatoria de 489 participantes. Se realizaron pruebas descriptivas-comparativas, de correlación, así como modelos de regresión logística ordinal. Los resultados muestran que las mujeres, los participantes con enfermedades respiratorias previas y quienes poseen estudios superiores son más propensos a autoprotegerse frente a la contaminación. Estos participantes tienden a ser más conscientes de la mala calidad del aire y perciben mejor la gravedad de sus efectos. Si bien este estudio muestra que las variables psicosociales son relevantes para comprender la adhesión a conductas de autoprotección, cada una de estas conductas debe entenderse de forma diferenciada, ya que los factores determinantes varían en cada una de ellas.

Key words: self-protective behaviors, risk perception, air quality, health

\begin{abstract}
The medium cities of southern Chile suffer critical episodes every winter due to emission of large quantities of $\mathrm{PM}_{2.5}$ from wood-burning stoves and wood cookstoves. Policy makers have implemented atmospheric decontamination plans, which include awareness campaigns to promote self-protective behaviors such as wearing face masks or exercising only within walls. The objective of this study was to analyze the role of
\end{abstract}


psychosocial factors in the adoption of self-protective behaviors against air pollution in residents of Temuco and Padre Las Casas. A non-experimental, cross-sectional and correlational-scope study was carried out, based on a non-probabilistic sample of 489 participants. Descriptive-comparative and correlation tests were performed, as well as ordinal logistic regression models. The results show that women, participants with previous respiratory illnesses, and those with higher education are more likely to protect themselves against pollution. These participants tend to be more aware of poor air quality and better perceive the severity of its effects. Although this study shows that psychosocial variables are relevant to understanding adherence to self-protective behaviors, each of these behaviors must be understood differently, since the determining factors vary in each of them.

\section{INTRODUCCIÓN}

Según la Organización Mundial de la Salud (OMS), el $92 \%$ de la población mundial vive en lugares con niveles de contaminación del aire insalubres (OMS 2019). Se estima que la mala calidad del aire causa 8.9 millones de muertes prematuras por año (Burnett 2018). Las partículas atmosféricas finas $\left(\mathrm{MP}_{2.5}\right)$, formadas por elementos microscópicos, son uno de los contaminantes del aire más peligrosos, pues se pueden inhalar fácilmente causando serios problemas de salud (Díaz-Robles et al. 2018). Durante las últimas dos décadas, diversos estudios han documentado de manera consistente la relación entre $\mathrm{MP}_{2.5}$ y problemas de salud, entre los que se incluyen enfermedades respiratorias (Medina-Ramón et al. 2006, Dominici et al. 2006), cardiovasculares (Peng et al. 2009, Brook et al. 2010) y neurocognitivas (Guxens y Sunyer 2012); problemas en el embarazo (Bell et al. 2010), o cáncer (Unosson et al. 2013, Díaz-Robles et al. 2018). La investigación también demuestra que la contaminación del aire puede generar efectos sociales negativos, como el aumento de las tasas de suicidio (Casas et al. 2017, Kim et al. 2018, Lee et al. 2018), el ausentismo escolar (Currie et al. 2009, Liu y Salvo 2018) o la disminución de la productividad laboral (Chang et al. 2016, Aragón et al. 2017).

Chile es uno de los países más afectados por la contaminación del aire por $\mathrm{MP}_{2.5}$ en América Latina. Siete de las 20 ciudades más contaminadas del continente son chilenas, incluyendo la más contaminada, Coyhaique, la cual, con una concentración media de $\mathrm{MP}_{2.5}$ anual de $64 \mu \mathrm{g} / \mathrm{m}^{3}$, supera ampliamente la norma anual establecida por la OMS de $10 \mu \mathrm{g} / \mathrm{m}^{3}$ (OMS 2019). La mayor parte de estas ciudades pertenecen a la zona centro-sur del país y no incluyen la capital, Santiago de Chile. Las ciudades intermedias de la mitad sur del país se caracterizan por sus inviernos fríos, húmedos y contaminados por el humo de leña. La precariedad de buena parte del parque de viviendas (Cortés y Ridley 2013, Schueftan y González 2015), el elevado precio de los combustibles alternativos y otros factores culturales (Boso et al. 2020a) o afectivos (Boso et al. 2020b) explican el origen de este problema socioambiental. Se estima que prácticamente el $80 \%$ de los hogares emplea leña como fuente de energía para calefacción o cocina en el sur del Chile urbano y más del $95 \%$ en los sectores rurales (ME 2015). Tanto es así que, en esta región, las estufas a leña son responsables de un $82 \%$ a $94 \%$ de las emisiones anuales de partículas atmosféricas gruesas $\left(\mathrm{MP}_{10}\right)$ y finas $\left(\mathrm{MP}_{2.5}\right)$, respectivamente.

Las comunas de Temuco y Padre Las Casas fueron declaradas zonas saturadas por contaminación de $\mathrm{MP}_{10}$ en 2005 y de $\mathrm{MP}_{2.5}$ en 2013, tras superar los umbrales permitidos por la legislación del país durante tres años consecutivos (Armijo et al. 2013). Frente a esta situación, el Ministerio del Medio Ambiente (MMA 2014) lideró la implementación del Plan de Descontaminación Ambiental que ha servido de modelo en el resto de ciudades del sur del país, aquejadas por dicha problemática. El plan combina medidas de regulación y restricción del uso de leña en periodos de emergencia sanitaria, con incentivos al recambio de estufas y subvenciones para la mejora de la calidad de las viviendas. El Ministerio del Medio Ambiente ha establecido algunas recomendaciones para la población (MMA 2019a) como el uso de mascarillas, especialmente en los grupos de riesgo como adultos mayores, niños, embarazadas y enfermos crónicos. Por su parte, el Ministerio de Salud (Minsal 2015) recomienda que en días de alerta y preemergencia ambiental se realice actividad física preferentemente en intramuros y en lugares alejados del alto tráfico vehicular. Si bien, los sujetos sanos pueden realizar todo tipo de actividad física manteniendo los niveles que realizan usualmente, los grupos sensibles (con enfermedades cardiovasculares, pulmonares, asma, niños y adolescentes, adultos mayores y embarazadas) 
deben realizar actividad física de intensidad ligera a moderada, con tiempos de recuperación prolongados, evitando esfuerzos físicos extendidos o muy intensos. En los días de emergencia se recomienda no realizar esfuerzos físicos, suspendiendo toda actividad física fuera de las labores habituales; asimismo, se sugiere que los grupos sensibles permanezcan en lugares intramuros ya que al realizar actividades físicas la intensidad respiratoria incrementa, al igual que los riesgos de salud. Pese a las recomendaciones, la adherencia a las conductas de autoprotección por parte de la población del sur de Chile parece muy variable y ha sido relativamente poco estudiada (Álvarez y Boso 2018).

\section{REVISIÓN DE LA LITERATURA}

Diversas dimensiones actitudinales son consideradas relevantes para comprender las reacciones del público frente a la contaminación del aire. Las conductas de autoprotección suelen definirse como las acciones que las personas llevan a cabo con la creencia de que su realización contribuirá al propio bienestar. La finalidad de la conducta autoprotectora es reducir la propia exposición sin necesariamente contribuir a la mitigación de la contaminación del aire (Zehaie 2009). A pesar de que las autoridades sanitarias de las ciudades afectadas por la mala calidad del aire suelen realizar recomendaciones, en general sólo una minoría de la población desarrolla acciones preventivas de manera voluntaria (Boso et al. 2020a) o acciones para la detección de enfermedades en fases asintomáticas (Qin et al. 2018). Estudios realizados en diversos contextos culturales apuntan a que en condiciones normales se suele apreciar una baja adherencia a las recomendaciones para evitar, reducir o reprogramar actividades de ocio o incluso realizarlas en interiores, con el objetivo de protegerse frente a la contaminación (Cai y He 2016, D'Antoni et al. 2017). Sin embargo, admitiendo esta tendencia general, la investigación social muestra que existen diferencias significativas entre diferentes tipos de acciones de protección.

Por ejemplo, los resultados de estudios realizados en Estados Unidos y China muestran que la actividad física al aire libre disminuye cuando aumenta la contaminación del aire por $\mathrm{MP}_{2.5}$ (Qian et al. 2016, Hu et al. 2017, Yu et al. 2019), registrándose una reducción promedio del ejercicio de 32.45 min semanales en algunos casos (Yu et al. 2017). Otras investigaciones han alertado que este cambio de comportamiento no implica necesariamente un estilo de vida más saludable, ya que a veces deriva en un aumento de conductas sedentarias (Ma et al. 2018). Asimismo, otros hábitos cotidianos pueden alterarse. Algunas personas cambian sus patrones de recreación y relaciones interpersonales, o bien postergan o cancelan sus actividades al aire libre en días de alta contaminación debido a la preocupación por los efectos nocivos que ésta pueda tener sobre su salud (Qian et al. 2016, Ma et al. 2018). Johnson (2012) y Ma et al. (2018) han observado una tendencia a refugiarse en el hogar cuando los pronósticos oficiales de contaminación son negativos y la fuente de información es confiable, por ejemplo, si el mensaje no contradice otras señales (e.g., información sensorial). Además, el estudio de Johnson (2012) en Nueva Jersey (EE.UU.), mostró que un $71 \%$ de los participantes permanecía en casa con las ventanas cerradas en días de alta contaminación del aire, como medida de autoprotección.

El uso de mascarillas con filtro es una de las medidas más efectivas para combatir la contaminación del aire, ya que permite bloquear las partículas contaminantes que se respiran y proteger el sistema respiratorio (Zhang y Mu 2018). Zhou et al. (2018) revelan que la efectividad de las mascarillas con microventilación es $>99.3 \%$ para el $\mathrm{MP}_{2.5}$, siendo altamente recomendadas con niveles superiores a $70 \mu \mathrm{g} / \mathrm{m}^{3}$ (Chung et al. 2019). Sin embargo, hasta la crisis del Covid-19 el uso de mascarillas era minoritario en muchos países. El escaso apoyo social asociado con el manejo de la mascarilla puede interferir con esta conducta, especialmente en algunos contextos culturales en los que existen ciertos prejuicios. En un estudio realizado en España, Sala et al. (2017) observaron que, antes de la pandemia del Covid-19, las personas no se planteaban su uso debido a las emociones de vergüenza y el temor a la burla social. En cambio, en China las mascarillas contra $\mathrm{MP}_{2.5}$ ganaron popularidad desde una serie de eventos que fueron apodados como "airpocalypse", generándose un aumento del $50.4 \%$ del uso total de mascarillas y un $70.6 \%$ de las mascarillas anti-MP 2.5 (Zhang y $\mathrm{Mu}$ 2018). Se prevee que la experiencia de la gestión del Covid-19 implique un aumento del uso de mascarillas en países tradicionalmente menos propensos a su empleo.

Finalmente, existen medidas que implican un costo económico mayor, como mudarse a áreas no contaminadas. De acuerdo con Evans y Jacobs (1981), la ubicación de la vivienda o la decisión de cambiar de residencia (a otra ciudad o sector) pueden basarse en los niveles de contaminación presentes en la zona. Sin embargo, las personas que viven en zonas 
más contaminadas suelen tener ingresos menores, hecho que dificulta este tipo de decisiones (Sun et al. 2017). En un estudio realizado en Barcelona, España, Sala et al. (2014) descubrieron que los participantes consideran una opción muy radical cambiar de barrio como resultado de la contaminación. En definitiva, la evidencia indica que mudarse sería interpretado como último recurso para protegerse de la contaminación (Evans y Jacobs 1981, Sala et al. 2014, Sun et al. 2017).

Varias dimensiones actitudinales suelen considerarse relevantes para comprender la adopción de conductas de autoprotección frente a la contaminación. La investigación psicológica y sociológica suele dar cuenta de la percepción de la calidad del aire, la percepción de riesgo y los niveles de preocupación y conciencia, como variables claves (Saksena 2011, Oltra y Sala 2015, Oltra et al. 2017). Por ejemplo, diversos estudios muestran que, a peor percepción de la calidad del aire del entorno, mayor suele ser la adopción de conductas protectoras (Semenza et al. 2008, Laumbach et al. 2015, Oltra y Sala 2016). Otras dimensiones, principalmente estudiadas en estudios epidemiológicos, son la irritación y ansiedad generadas por la contaminación (Jacquemin et al. 2007), la sensación de pérdida de calidad de vida (Deguen et al. 2012) o la percepción sensorial (Langer et al. 2017). La búsqueda de información o la atención a los avisos de las autoridades sanitarias también han sido objeto de estudio de la investigación psicosocial en las últimas décadas (Johnson 2002, Henry y Gordon 2003, Neidell 2006, Semenza et al. 2008, Noonan 2011, Wells et al. 2012, Oltra y Sala 2016, Gurajala y Matthews 2018). Lin et al. (2016) plantean, por ejemplo, que los jóvenes (quienes sacan mayor provecho de tecnologías como Instagram, Facebook y Twitter para informarse y comunicarse) suelen hablar más de temas relacionados con la contaminación en su círculo social cercano. Estos factores incrementan su probabilidad de sentirse afectados por las consecuencias de la mala calidad del aire y, consecuentemente, los orienta mejor en sus reacciones frente a la contaminación (Lin et al. 2016, Sun et al. 2017).

El efecto de las variables sociodemográficas en la percepción de la contaminación y la adopción de medidas de protección también han sido foco de estudio de la investigación social. La edad, el sexo, el nivel educacional o los antecedentes de enfermedades respiratorias crónicas suelen ser variables destacadas por la literatura, aunque no existe un consenso total acerca de su efecto. Por ejemplo, Lissåker et al. (2014) reportan que las personas de 50 a 60 años de edad tienen mayor probabilidad de protegerse, especialmente si son mujeres. Sin embargo, otros estudios muestran que son los participantes jóvenes y de mediana edad quienes tienden a ser más conscientes y protegerse (Fischer et al. 1991). Las mujeres parecen mostrar mayores niveles de conocimiento acerca de la contaminación en comparación con los hombres en algunos estudios (Finucane et al. 2000, Johnson 2002, Liao et al. 2015), pero esa tendencia no se observa en todas las investigaciones (Álvarez y Boso 2018). Por otro lado, el nivel educacional parece ser una variable relevante, por cuanto las personas con educación universitaria o superior tienen más probabilidades de adoptar estas conductas (Qian et al. 2016). Sin embargo, la literatura sobre justicia ambiental muestra que, en ocasiones, las personas que pertenecen a minorías étnicas y viven en barrios pobres pueden percibir mejor los riesgos asociados con la contaminación, dado que son más conscientes de la precariedad de su entorno físico (Cutter 1981, Flynn et al. 1994, Finucane et al. 2000). Por otro lado, los resultados respecto al efecto de las enfermedades crónicas previas parece ser consistente en general. Las personas que están afectadas directa o indirectamente por estos problemas de salud, tienden a adoptar conductas de autoprotección con mayor frecuencia (Wells et al. 2012, Oltra y Sala 2016, Barría et al. 2016).

El objetivo de este estudio es comprender si los factores psicosociales y sociodemográficos predicen las conductas de autoprotección en dos ciudades del sur de Chile, Temuco y Padre Las Casas, gravemente afectadas por la contaminación del aire. El artículo plantea seis modelos predictivos de conductas de autoprotección frente a la contaminación del aire, que han mostrado se relevancia en la literatura especializada pero no habían sido planteados previamente en la región.

\section{MÉTODO}

\section{Área de estudio}

Temuco y Padre Las Casas son dos ciudades ubicadas en la región de La Araucanía, zona sur de Chile. Ambas ciudades forman una conurbación en una superficie de $864.7 \mathrm{~km}^{2}$, estando separadas sólo por el río Cautín, con un total de 358541 habitantes (INE 2017). Estas ciudades se ubican en una zona de bajas temperaturas, las cuales varían anualmente entre -4 y $31^{\circ} \mathrm{C}$, aproximadamente, con un promedio de $7{ }^{\circ} \mathrm{C}$. Durante los meses de invierno es recurrente observar temperaturas inferiores a $0{ }^{\circ} \mathrm{C}$ (Meteored 
2019, Weather Spark 2019). Igualmente, cuentan con características topográficas que predisponen a altos niveles de MP, al ubicarse en áreas de planicie y en la ribera del río Cautín, la cual en épocas invernales genera una alta presencia de neblina, empeorando la calidad del aire (MMA 2015).

IQAir (2019) posiciona a Padre Las Casas como la tercera ciudad más contaminada de Chile con relación a $\mathrm{MP}_{2.5}$, sólo superada por Coyhaique y Osorno. Pese a las medidas tomadas por el MMA durante 2018, se detectaron 31 episodios de emergencia en Temuco $\left(\mathrm{MP}_{2.5}>170 \mu \mathrm{g} / \mathrm{m}^{3}\right)$, concentrando los episodios más críticos en las horas de la tarde-noche (MMA 2018, 2019b).

\section{Diseño}

Este estudio se realizó con un diseño no experimental transversal (Hernández et al. 2014, Corona 2016) por medio de la aplicación de una encuesta, a fin de correlacionar las variables de estudio con variables sociodemográficas (Díaz-Narváez y Calzadilla 2016).

\section{Participantes y muestra}

La información se obtuvo por medio de un muestreo no probabilístico por conveniencia. Durante el invierno de 2017 se seleccionaron un mínimo de 50 viviendas en Padre Las Casas y en cada uno de los siete macrosectores urbanos de Temuco. Cabe señalar que las restricciones al uso de estufas de leña no operan en todos los sectores de la ciudad, por lo que la diversidad espacial de la muestra garantizó que se tuvieran controlados los factores institucionales. Los participantes debían tener más de 18 años y manejar el sistema de calefacción que se utiliza en su hogar (ya sea de forma exclusiva o compartida). En primer lugar, en cada sector se seleccionaron, por un lado, hogares que se hubieran adherido a la política de recambio de estufas $(21.5 \%) \mathrm{y}$, por otro lado, hogares que emitieran humos visibles. En segundo lugar, el estudio se completó con viviendas elegidas de forma aleatoria en las mismas calles o manzanas en las que se había entrevistado a personas según los dos criterios anteriores (48.5\%). De esta forma, la muestra final quedó compuesta por 489 personas residentes de Temuco y Padre Las Casas (Cuadro I). La edad de los participantes varía de 18 a 89 años $(\mathrm{M}=43.42 ; \mathrm{DS}=18.64)$

\section{Procedimiento y materiales}

Los participantes fueron encuestados en sus hogares, ubicados en diversas calles y barrios de diferentes macrosectores de Temuco y Padre Las Casas.
CUADRO I. CARACTERÍSTICAS SOCIODEMOGRÁFICAS DE LOS PARTICIPANTES.

\begin{tabular}{lr}
\hline Variables & $\mathrm{n}(\%)$ \\
\hline Sexo & \\
Hombre & $190(38.9)$ \\
Mujer & $298(61.1)$ \\
Educación & \\
Básico o menor & $57(11.7)$ \\
Media & $138(28.2)$ \\
Superior & $294(60.1)$ \\
Pertenece a pueblos originarios & \\
Sí & $78(16.0)$ \\
No & $411(84.0)$ \\
Tiene enfermedades respiratorias & \\
Sí & $63(12.9)$ \\
No & $424(87.1)$ \\
Sistema de calefacción & \\
Leña & $320(65.4)$ \\
Parafina & $58(11.9)$ \\
Otros (gránulos, electricidad) & $108(22.1)$ \\
\hline
\end{tabular}

Los objetivos y resguardos éticos del estudio fueron explicados a cada participante. Quienes aceptaron participar firmaron un consentimiento informado que detallaba aspectos como la confidencialidad y uso responsable de los datos.

La encuesta aplicada aborda diversas temáticas relativas a la percepción sobre la calidad del aire en Temuco y Padre Las Casas. Las variables de estudio se midieron por medio de preguntas de respuesta múltiple. Para la percepción de riesgo, se utilizaron las siguientes preguntas: 1) ¿cree que, en general, los efectos de la contaminación del aire sobre la salud de las personas son: $1=$ leves a 3 = graves?; y 2) ¿cree que, en general los efectos de la contaminación del aire sobre la salud de las personas son: $1=$ a corto plazo a 3 = a largo plazo? También se consultó lo siguiente: ¿durante el último invierno, a causa de la contaminación del aire, ha pensado que su calidad de vida disminuía? $(1=$ sí y $2=$ no). Por su parte, la percepción de la calidad del aire se midió a través de dosítems tipo Likert: 1) ¿cómo evaluaría la calidad del aire en Temuco? $(1=$ muy mala a $5=$ muy buena); y (2) ¿suele usted prestar atención a la calidad del aire que respira al salir de casa o del trabajo? $(1=$ nunca a $4=$ siempre). Igualmente se consultó lo siguiente: ¿ha discutido o comentado en el último mes con amigos y/o conocidos sobre la contaminación del aire? $(1=$ sí y $2=$ no $)$.

Para medir las conductas autoprotectoras, se consultó a los participantes sobre la frecuencia con 
la cual realizaron alguna de las siguientes acciones a causa de la contaminación del aire durante el último invierno: a) cambiar sus actividades de ocio, b) evitar realizar ejercicio físico al aire libre, c) permanecer en casa, d) evitar abrir las ventanas, e) utilizar mascarilla y f) pensar en mudarse de casa. En cada caso, las opciones de respuesta fueron de $1=$ nunca a $4=$ siempre.

Adicionalmente, se recolectaron datos sociodemográficos de edad, sexo, nivel educacional, pertenencia a pueblos originarios yo otros datos de interés, incluyendo el sistema de calefacción utilizado o si alguien en el hogar tiene enfermedades crónicas. Responder la encuesta tomaba un tiempo aproximado de 20 minutos.

\section{Análisis de datos}

Para el análisis de datos se utilizó el software estadístico SPSS 24. En un tratamiento preliminar de los datos, se revisaron datos perdidos y valores atípicos. Ninguna de las variables de estudio cumplió con el supuesto de normalidad $(\mathrm{K}-\mathrm{S}<.05)$, por lo que se trabajó con pruebas no paramétricas.

Inicialmente se efectuaron estadísticos descriptivos de las conductas protectoras. Posteriormente se realizaron pruebas de comparación con base en variables sociodemográficas por medio de las pruebas U de Mann-Whitney y Kruskal-Wallis. Finalmente, para revisar la asociación entre las variables independientes y las conductas de autoprotección ante la contaminación del aire, se utilizaron modelos de regresión ordinal (Long 1997), con enlace logit y con revisión del supuesto de líneas paralelas. Este análisis permite predecir la relación entre una variable dependiente de nivel ordinal con diversas variables independientes. Las variables independientes consideradas para cada modelo fueron sexo, edad, nivel educacional, enfermedades respiratorias previas, plazo y gravedad de los efectos de la contaminación en la salud, efecto de la contaminación en la calidad de vida, percepción de la calidad del aire, hablar con amigos sobre la contaminación y prestar atención a la calidad del aire.

En los casos en que no se cumplió el supuesto de líneas paralelas, se optó por modificar el enlace logit a un enlace log-log negativo (sesgo hacia la izquierda) o log-log complementario (sesgo hacia la derecha) según correspondíaa a la distribución de la variable. Cuando los datos no se ajustaron bien al modelo ordinal se optó por una regresión logística multinomial, ya que es robusta frente al incumplimiento del supuesto de líneas paralelas (Hosmer y Lemeshow 1989).

\section{RESULTADOS}

Respecto de las conductas autoprotectoras que los participantes adoptaron frente a la contaminación del aire, se aprecia que permanecer en casa $(\mathrm{M}=2.92$; $\mathrm{DS}$ $=1.06)$ y evitar abrir las ventanas $(\mathrm{M}=2.80 ; \mathrm{DS}=$ 1.14) fueron las acciones que los encuestados realizan con mayor frecuencia. Cambiar actividades de ocio $(\mathrm{M}=2.30 ; \mathrm{DS}=1.07)$ y evitar realizar ejercicio físico al aire libre $(\mathrm{M}=2.61 ; \mathrm{DS}=1.22)$ se reportaron de forma medianamente frecuente. En contraste, el uso de mascarilla $(\mathrm{M}=1.20 ; \mathrm{DS}=0.58)$ y considerar mudarse de casa $(\mathrm{M}=1.44$; $\mathrm{DS}=0.89)$ fueron las conductas de protección que menos dijeron realizar frente a la contaminación del aire.

Al comparar las conductas protectoras según variables sociodemográficas (Cuadro II), se observan diferencias significativas según sexo, siendo las mujeres quienes más reportan cambiar sus actividades de ocio $(U=24982.00 ; p<0.05)$, permanecer en casa $(U=24799.50 ; p<0.05)$ y utilizar mascarilla $(U=26173.50 ; p<0.05)$. Igualmente, se observan diferencias respecto a la edad: los menores de 60 años mencionan realizar más a menudo las conductas de cambiar actividades de ocio $(U=18581.00 ; p<$ $0.05)$ y evitar ejercicio al aire libre $(U=18376.00$; $p<0.05$ ), en comparación con quienes tienen $60 \mathrm{o}$ más años. Se observa que quienes afirman tener una enfermedad respiratoria reportan cambiar más sus actividades de ocio $(U=11054.50 ; p<0.05)$, evitar ejercicio al aire libre $(U=10474.50 ; p<0.05)$ y permanecer en casa $(U=10493.00 ; p<0.01)$, en comparación con población saludable. Asimismo, quienes tienen enfermedades respiratorias reportan más el uso de mascarilla $(U=10997.50 ; p<0.001)$ o la consideración de mudarse de casa $(U=9723.00$; $p<0.001)$.

Por otro lado, al observar diferencias en las conductas autoprotectoras según el nivel educacional (Cuadro III), se evidencian diferencias significativas: quienes tienen estudios superiores, informan cambiar más frecuentemente sus actividades de ocio $\left(\chi^{2}=9.739 ; p<0.01\right)$ o evitar ejercicio al aire libre $\left(\chi^{2}=8.515 ; p<0.05\right)$ en comparación con personas que tienen una educación básica o menor.

Se realizó una serie de regresiones ordinales para construir los modelos predictores de las conductas autoprotectoras frente a la contaminación del aire. En el caso de la variable 'cambiar actividades de ocio', no se cumple el supuesto de líneas paralelas $(p=0.01)$. En consecuencia, se optó por un modelo multinomial que no presenta restricciones ante el incumplimiento de este supuesto. El modelo de las 
CUADRO II. COMPARACIÓN DE CONDUCTAS DE AUTOPROTECCIÓN SEGÚN SEXO, EDAD Y ENFERMEDADES PREVIAS.

\begin{tabular}{lccc}
\hline \multirow{2}{*}{ Sexo } & Mujer & Hombre & \multirow{2}{*}{ U } \\
\cline { 2 - 3 } & M (DS) & M (DS) & \\
Cambiar actividades de ocio & $2.40(1.10)$ & $2.20(1.03)$ & $24982.00^{*}$ \\
Evitar ejercicio al aire libre & $2.62(1.25)$ & $2.61(1.19)$ & 27402.00 \\
Permanecer en casa & $3.00(1.05)$ & $2.79(1.08)$ & $24799.50^{*}$ \\
Evitar abrir ventanas & $2.85(1.10)$ & $2.74(1.20)$ & 26810.00 \\
Utilizar mascarilla & $1.22(0.59)$ & $1.15(0.59)$ & $26173.50^{*}$ \\
Mudarse de casa & $1.44(0.90)$ & $1.44(0.89)$ & 27559.00 \\
\hline Edad & $<60$ & $\geq 60$ & \\
\hline Cambiar actividades de ocio & $2.39(1.04)$ & $2.11(1.18)$ & $18581.00^{*}$ \\
Evitar ejercicio al aire libre & $2.70(1.18)$ & $2.35(1.31)$ & $18376.00^{*}$ \\
Permanecer en casa & $2.90(1.03)$ & $3.00(1.13)$ & 19925.00 \\
Evitar abrir ventanas & $2.84(1.13)$ & $2.72(1.18)$ & 20638.50 \\
Utilizar mascarilla & $1.18(0.57)$ & $1.24(0.65)$ & 21105.50 \\
Mudarse de casa & $1.46(0.91)$ & $1.39(0.85)$ & 20926.00 \\
\hline Enfermedades respiratorias & Sí & No & \\
\hline Cambiar actividades de ocio & $2.60(1.14)$ & $2.28(1.06)$ & $11054.50^{*}$ \\
Evitar ejercicio al aire libre & $3.02(1.10)$ & $2.55(1.23)$ & $10474.50^{*}$ \\
Permanecer en casa & $3.24(0.99)$ & $2.87(1.06)$ & $10493.00^{* *}$ \\
Evitar abrir ventanas & $2.71(1.17)$ & $2.82(1.14)$ & 12650.00 \\
Utilizar mascarilla & $1.44(0.84)$ & $1.16(0.53)$ & $10997.50^{* * *}$ \\
Mudarse de casa & $1.94(1.19)$ & $1.37(0.82)$ & $9723.00^{* * *}$ \\
\hline & & & \\
\hline
\end{tabular}

$* p<0.05 ; * * p<0.01 ; * * p<0.001$. M: media; DS: desviación estándar.

CUADRO III. COMPARACIÓN DE CONDUCTAS DE AUTOPROTECCIÓN SEGÚN ${ }^{\circ}$ NIVEL EDUCACIONAL.

\begin{tabular}{lccccc}
\hline \multirow{2}{*}{ Conducta protectora } & $\begin{array}{c}1 \\
\text { (básica o menor) }\end{array}$ & $\begin{array}{c}2 \\
\text { (media) }\end{array}$ & $\begin{array}{c}3 \\
\text { (superior) }\end{array}$ & Post-hoc \\
\cline { 2 - 6 } & M (DS) & M (DS) & M (DS) & $\chi^{2}$ & U \\
\hline Cambiar actividades de ocio & $1.97(1.05)$ & $2.28(1.14)$ & $2.41(1.04)$ & $9.739^{* *}$ & $1<3$ \\
Evitar ejercicio al aire libre & $2.30(1.29)$ & $2.44(1.29)$ & $2.75(1.15)$ & $8.515^{*}$ & $1<3$ \\
Permanecer en casa & $3.12(1.07)$ & $2.96(1.12)$ & $2.86(1.06)$ & 5.687 & \\
Evitar abrir ventanas & $2.68(1.15)$ & $2.81(1.17)$ & $2.82(1.13)$ & 0.763 & \\
Utilizar mascarilla & $1.21(0.59)$ & $1.26(0.72)$ & $1.16(0.51)$ & 1.487 & \\
Mudarse de casa & $1.32(0.71)$ & $1.50(0.97)$ & $1.44(0.89)$ & 0.779 & \\
\hline
\end{tabular}

$* p<0.05 ; * * p<0.01 ; * * * p<0.001$. M: media; DS: desviación estándar.

conductas de ocio (Cuadro IV) presenta un ajuste significativo $\left(\chi^{2}=218.95 ; p<0.001\right)$, con una bondad de ajuste adecuado según los indicadores de Pearson $\left(\chi^{2}=1018.73 ; p=0.750\right)$ y devianza $\left(\chi^{2}=853.94 ; p\right.$ $=1.000)$, y un pseudo $\mathrm{R}^{2}$ de Nagelkerke $=0.398$. El modelo indica que aquellos sujetos que más dicen cambiar sus actividades de ocio son quienes perciben los efectos de la contaminación del aire sobre la salud como graves y a corto plazo $(\beta=-0.822 ; p=0.043)$.
Asimismo, quienes indicaron cambiar sus actividades de ocio, fueron las personas que evaluaron la calidad del aire como muy mala y que prestan atención a ésta $(\beta=1.858 ; p<0.001)$. En cuanto a variables sociodemográficas, las mujeres tienen una probabilidad mayor de cambiar sus actividades de ocio $(\beta=$ $-0.932 ; p=0.007$ ). Por otro lado, quienes tienen un nivel de estudios básicos o menores, tienen menos probabilidades de realizar esta conducta $(\beta=1.251$; 
CUADRO IV. REGRESIÓN MULTINOMIAL DE LA CONDUCTA CAMBIAR ACTIVIDADES DE OCIO.

\begin{tabular}{|c|c|c|c|c|c|c|}
\hline \multirow{2}{*}{ Variables } & \multicolumn{2}{|c|}{ Casi nunca } & \multicolumn{2}{|c|}{ A menudo } & \multicolumn{2}{|c|}{ Siempre } \\
\hline & Coeficiente & $p$ & Coeficiente & $p$ & Coeficiente & $p$ \\
\hline Intercepción & 37.245 & 0.994 & 18.075 & 0.000 & 34.513 & 0.994 \\
\hline \multicolumn{7}{|l|}{ Sexo } \\
\hline$(\mathrm{x}=1)$ Mujer & -0.725 & 0.052 & -0.650 & 0.107 & -0.932 & 0.007 \\
\hline$(x=2)$ Hombre & Ref. & & Ref. & & Ref. & \\
\hline \multicolumn{7}{|l|}{ Edad } \\
\hline$(x=1)<60$ & -0.076 & 0.849 & -0.493 & 0.309 & 0.537 & 0.159 \\
\hline$(x=2) \geq 60$ & Ref. & & Ref. & & Ref. & \\
\hline \multicolumn{7}{|l|}{ Educación } \\
\hline$(\mathrm{x}=1)$ Básica o menor & 1.251 & 0.040 & 0.860 & 0.205 & 0.642 & 0.284 \\
\hline$(\mathrm{x}=2)$ Media & 0.579 & 0.130 & -0.415 & 0.349 & 0.256 & 0.466 \\
\hline$(\mathrm{x}=3)$ Superior & Ref. & & Ref. & & Ref. & \\
\hline \multicolumn{7}{|l|}{ Enfermedades respiratorias } \\
\hline$(\mathrm{x}=1)$ Sí & -0.244 & 0.591 & -0.996 & 0.082 & -0.367 & 0.358 \\
\hline$(x=2)$ No & Ref. & & Ref. & & Ref. & \\
\hline \multicolumn{7}{|l|}{ Gravedad de efectos } \\
\hline$(\mathrm{x}=1)$ Graves & -17.443 & $<0.001$ & -18.130 & $<0.001$ & -16.314 & $<0.001$ \\
\hline$(x=2)$ Medios & -16.125 & $<0.001$ & -16.794 & $<0.001$ & -15.782 & $<0.001$ \\
\hline$(x=3)$ Leves & Ref. & & Ref. & & Ref. & \\
\hline \multicolumn{7}{|l|}{ Peor calidad de vida } \\
\hline$(\mathrm{x}=1) \mathrm{Sí}^{\prime}$ & -0.837 & 0.027 & -0.259 & 0.533 & 0.350 & 0.351 \\
\hline$(x=2)$ No & Ref. & & Ref. & & Ref. & \\
\hline \multicolumn{7}{|l|}{ Evaluación calidad del aire } \\
\hline$(\mathrm{x}=1)$ Muy Mala & -18.718 & 0.997 & -1.404 & 0.005 & -17.864 & 0.997 \\
\hline$(x=2)$ Mala & -18.127 & 0.997 & -0.289 & 0.562 & -17.642 & 0.997 \\
\hline$(\mathrm{x}=3)$ Regular & -18.663 & 0.997 & -0.935 & 0.562 & -18.064 & 0.997 \\
\hline$(x=4)$ Buena & -18.464 & 0.997 & -18.925 & 0.996 & -18.212 & 0.997 \\
\hline$(x=5)$ Muy Buena & Ref. & & Ref. & & Ref. & \\
\hline \multicolumn{7}{|l|}{ Hablar con amigos } \\
\hline$(x=1)$ Sí & -0.761 & 0.063 & -0.171 & 0.710 & -0.193 & 0.632 \\
\hline$(x=2)$ No & Ref. & & Ref. & & Ref. & \\
\hline \multicolumn{7}{|l|}{ Atención calidad del aire } \\
\hline$(\mathrm{x}=1)$ Nunca & 1.120 & 0.135 & 0.399 & 0.677 & 0.251 & 0.746 \\
\hline$(x=2)$ Casi nunca & 0.770 & 0.231 & 0.932 & 0.177 & 0.155 & 0.812 \\
\hline$(x=3)$ A menudo & 0.597 & 0.135 & 1.858 & 0.000 & 0.608 & 0.099 \\
\hline$(x=4)$ Siempre & Ref. & & Ref. & & Ref. & \\
\hline \multicolumn{7}{|l|}{ Plazo de los efectos } \\
\hline$(\mathrm{x}=1)$ A corto plazo & -0.822 & 0.043 & 0.079 & 0.864 & -0.330 & 0.360 \\
\hline$(x=2)$ A mediano plazo & 0.588 & 0.161 & 1.184 & 0.008 & 1.038 & 0.009 \\
\hline$(\mathrm{x}=3)$ A largo plazo & Ref. & & Ref. & & Ref. & \\
\hline
\end{tabular}

La categoría de referencia es "Nunca".

$p=0.040$ ). Por último, se observa que quienes casi nunca cambian sus actividades de ocio tienen menos probabilidades de ver sus vidas afectadas a causa de la contaminación ( $\beta=-0.837 ; p=0.027$ ) en comparación con quienes nunca realizan esta conducta.

Los modelos para las otras cinco conductas autoprotectoras cumplieron el supuesto de líneas paralelas, por lo que se presentan en una serie de regresiones ordinales (Cuadro V). El modelo de la conducta 'evitar hacer ejercicio', indica un ajuste de modelo significativo $\left(\chi^{2}=78.20 ; p<0.001\right)$, cumpliendo con el supuesto de líneas paralelas $\left(\chi^{2}=\right.$ 48.23; $p=0.084)$. La bondad de ajuste es adecuada según los indicadores de Pearson $\left(\chi^{2}=1150.50 ; p\right.$ $=0.076)$ y devianza $\left(\chi^{2}=938.97 ; p=0.999\right)$, con un pseudo $\mathrm{R}^{2}$ de Nagelkerke $=0.165$. Se observa que los participantes que perciben como graves los efectos de la contaminación del aire en la salud ( $\beta$ $=0.463 ; p=0.043)$ tienen más probabilidades de evitar hacer ejercicio. Por otro lado, quienes nunca 
COMPORTAMIENTOS DE AUTOPROTECCIÓN FRENTE A LA CONTAMINACIÓN DEL AIRE

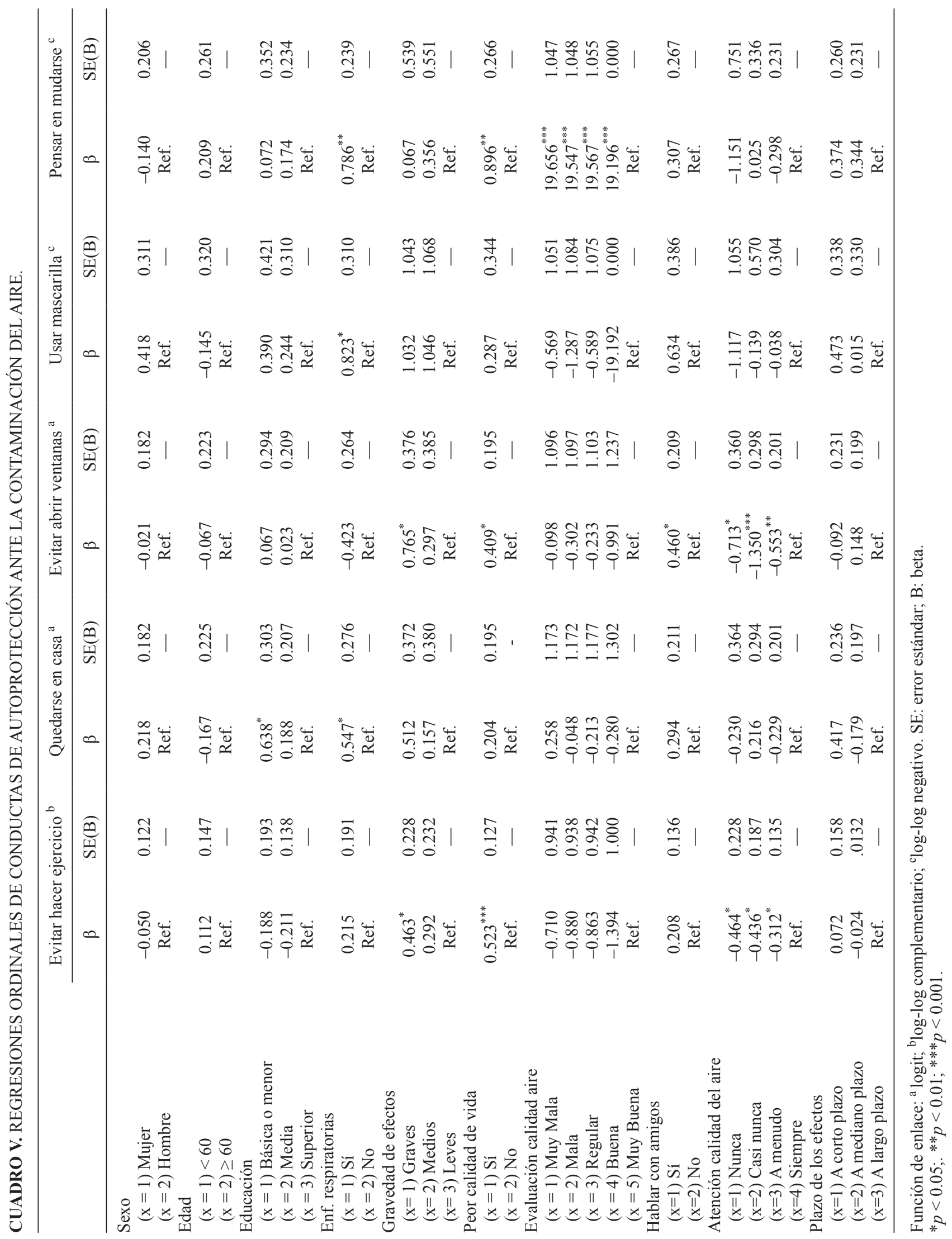


prestan atención a la calidad del aire $(\beta=-0.464$; $p=0.042)$ tenderán a hacer más ejercicio en comparación con quienes siempre le prestan atención. Quienes han visto empeorar su calidad de vida debido a la contaminación $(\beta=0.523 ; p<0.001)$ son quienes más reportaron evitar realizar ejercicio al aire libre.

El modelo de la conducta de 'permanecer en casa' es significativo $\left(\chi^{2}=50.134 ; p<.001\right)$ y cumple con la prueba de líneas paralelas $\left(\chi^{2}=23.39 ; p=0.948\right)$. Su bondad de ajuste es adecuada según los indicadores de Pearson $\left(\chi^{2}=1061.74 ; p=0.717\right)$ y devianza $\left(\chi^{2}=908.72 ; p=1.000\right)$ y tiene un pseudo $\mathrm{R}^{2}$ de Nagelkerke $=0.109$. En este caso, se puede apreciar que aquellos que presentaron mayores posibilidades de permanecer en casa son quienes padecen alguna enfermedad respiratoria crónica previa $(\beta=0.547$; $p=0.047$ ). En cuanto al nivel educacional, se observa que aquellos que tienen una educación básica o menor tienden a quedarse más en casa $(\beta=0.638$; $p=0.035)$ en comparación con aquellos que tienen educación superior.

El modelo de 'evitar abrir las ventanas' presenta un ajuste significativo $\left(\chi^{2}=79.52 ; p<0.001\right)$, cumpliendo con el supuesto de líneas paralelas $\left(\chi^{2}\right.$ $=41.46 ; p=0.245)$, así como una bondad de ajuste adecuado según los indicadores de Pearson $\left(\chi^{2}=\right.$ $1076.81 ; p=0.547)$, devianza $\left(\chi^{2}=954.58 ; p=\right.$ $0.998)$ y un pseudo $R^{2}$ de Nagelkerke $=0.166$. En este caso se observa que quienes evitaron abrir las ventanas en el último invierno a causa de la contaminación son quienes percibieron que los efectos de la contaminación en la salud son graves $(\beta=0.765 ; p=$ 0.042). Asimismo, quienes discutieron o comentaron con amigos y/o conocidos sobre la contaminación del aire, así como aquellos que siempre prestaron atención a la calidad del aire al salir de casa o del trabajo mostraron más posibilidades de evitar abrir las ventanas $(\beta=0.460 ; p=0.028)$. También se observa que los participantes que han visto disminuida su calidad de vida debido a la contaminación tienen más probabilidades de realizar esta conducta $(\beta=$ $0.409 ; p=0.036$ ).

Respecto al uso de mascarilla durante el último invierno a causa de la contaminación, se obtiene un ajuste de modelo significativo $\left(\chi^{2}=39.55 ; p=<\right.$ 0.002 ), cumpliendo con el supuesto de líneas paralelas $\left(\chi^{2}=28.75 ; p=0.799\right)$, así como una bondad de ajuste adecuado según los indicadores de Pearson $\left(\chi^{2}=1009.98 ; p=0.951\right)$ y devianza $\left(\chi^{2}=339.31\right.$; $p=1.000$ ), $\mathrm{y}$ un pseudo $\mathrm{R}^{2}$ de Nagelkerke $=0.128$. Conforme a los análisis realizados, los datos encontrados revelaron que los sujetos que mostraron mayores posibilidades de usar mascarilla fueron quienes padecen alguna enfermedad respiratoria crónica $(\beta$ $=0.823 ; p=0.008$ ).

Finalmente, referente a pensar en mudarse de casa, se presenta un ajuste de modelo significativo $\left(\chi^{2}=50.88 ; p<0.001\right)$, que cumple con el supuesto de líneas paralelas $\left(\chi^{2}=16.70 ; p=0.997\right)$, muestra una bondad de ajuste adecuado según los indicadores de Pearson $\left(\chi^{2}=1052.35 ; p=0.721\right)$ y devianza $\left(\chi^{2}=556.40 ; p=1.000\right)$, con un pseudo $\mathrm{R}^{2}$ de Nagelkerke $=0.129$. Se observa que los sujetos con más probabilidad de pensar en cambiarse de casa a causa de la contaminación del aire son aquellos que tienen alguna enfermedad respiratoria crónica $(\beta=0.786$; $p=0.001$ ), así como aquellos que han pensado que su calidad de vida se ha visto disminuida $(\beta=0.896$; $p=0.001)$. De igual manera, quienes muestran más posibilidades de pensar en mudarse de casa son quienes han evaluado la calidad del aire en Temuco o Padre Las Casas como muy mala $(\beta=19.656$; $p<0.001)$ en comparación con quienes la consideran muy buena.

\section{DISCUSIÓN}

El objetivo de este estudio fue identificar el rol de los factores psicosociales en la adhesión de seis conductas de autoprotección frente a la contaminación del aire en dos ciudades con altos niveles de $\mathrm{MP}_{2.5}$ en Chile. Todas las conductas analizadas tienen carácter voluntario. Algunas, como evitar hacer ejercicio físico al aire libre o el uso de mascarillas, han sido ampliamente recomendadas por los ministerios de Medio Ambiente o Salud, en tanto que otras, como evitar abrir ventanas o mudarse de casa, no suelen estar tan presentes en los mensajes institucionales. La no obligatoriedad de las medidas analizadas les confiere un bajo seguimiento por parte de las autoridades responsables, pues carecen de una legislación específica y, por lo general, no se evalúa su nivel de adhesión. En este contexto, los estudios empíricos son claves para entender en qué condiciones las personas que viven en ciudades con mala calidad de aire se protegen a sí mismas.

Los resultados muestran que, en general, los participantes realizan dichas conductas, pero con una frecuencia diversa, presentando una mayor adhesión a evitar realizar ejercicio al aire libre, permanecer en casa y evitar abrir ventanas. El Ministerio de Salud ha realizado mayores esfuerzos en sus campañas de sensibilización, recomendando a las personas evitar la realización de actividades físicas durante los días de alta contaminación ambiental y desarrollar 
actividades intramuros (Minsal 2015). El énfasis en las políticas de comunicación de riesgo podría explicar una mayor interiorización de esta conducta. Por otro lado, permanecer en casa o evitar abrir ventanas son acciones con un bajo costo percibido por parte de la población, hecho que suele favorecer una mayor adhesión (Lindell y Perry 1992).

Las actividades con menos probabilidades de ocurrir son cambiar las actividades de ocio, el uso de mascarillas (la encuesta se realizó antes de la pandemia del Covid-19) y pensar en mudarse de casa. Estos resultados son coherentes con buena parte de la literatura especializada. El estudio de Johnson (2012), por ejemplo, mostró que los participantes no solían cambiar sus actividades de ocio como resultado de la contaminación del aire a pesar que la consideraran un problema. El bajo uso de mascarillas también había sido registrado en estudios cualitativos previos, que explicaron su infravaloración por las emociones de vergüenza y temor a la burla social que podía conllevar en determinados contextos culturales (Sala et al. 2017). A su vez, la opción de mudarse de casa suele considerarse radical e inaccesible para las personas con menores ingresos (Evans y Jacobs 1981, Sala et al. 2014, Sun et al. 2017). Nuestro estudio muestra que las personas con antecedentes de enfermedades crónicas y que perciben una disminución en su calidad de vida de la contaminación son las que se plantean el cambio de domicilio como estrategia de protección. Diversos estudios señalan que las personas cuya salud o la de sus familiares ha sido afectada a causa de la contaminación del aire tienden a ser más conscientes de la severidad del problema $\mathrm{y}$, consecuentemente, se protegen más (Molina et al. 2001, Barría et al. 2016, Qin et al. 2018). En el mismo sentido, nuestro estudio también encontró una relación entre la presencia de enfermedades crónicas en el hogar y una probabilidad mayor de usar mascarillas o quedarse en casa.

Con relación a los factores sociodemográficos, el género, la educación y en menor grado la edad parecen estar asociados a algunas conductas de protección. Los resultados indican que las mujeres tienden a cambiar más sus actividades de ocio, permanecer en casa y utilizar mascarilla para protegerse frente a la contaminación que los hombres. Este hallazgo es también consistente con los resultados de diversos estudios previos (Johnson 2012, Lissåker et al. 2014, Oltra y Sala 2016). De acuerdo con algunos autores, las mujeres son más sensibles a los efectos de la contaminación del aire, ya que sus alvéolos pulmonares presentan una mayor predisposición biológica para la deposición de las partículas finas en su estructura, lo cual provoca mayor riesgo de enfermedades pulmonares (Kim y Hu 1998, Oliveira et al. 2011). En este sentido, la mayor adhesión a conductas de protección podría explicarse como respuesta a un mayor riesgo objetivo, aunque otros factores culturales y de socialización podrían actuar como variables mediadoras. Los modelos de regresión muestran resultados mixtos en cuanto al efecto de la educación, que deben interpretarse de manera cautelosa. Se observa una tendencia de los participantes con menor nivel de estudios a no cambiar sus actividades de ocio a causa de la contaminación. Sin embargo, este mismo grupo muestra mayor probabilidad de permanecer en casa a causa de la contaminación del aire.

Existen dos posibles mecanismos que pueden ayudarnos a interpretar esta información. En primer lugar, los participantes con menos estudios suelen pertenecer a un estrato socioeconómico más bajo, que a su vez suele residir en los barrios más contaminados y estar expuesto a niveles altos de contaminación extra e intradomiciliaria; en este sentido, dicho grupo podría reaccionar a a la exposición cotidiana quedándose en casa. Estudios previos muestran que los sujetos con bajo nivel de estudios se protegen con base en la percepción propia de la calidad del aire en lugar de prestar atención a las alertas oficiales (Kan et al. 2008, D'Antoni et al. 2017). En segundo lugar, la tendencia a no cambiar las actividades de ocio podría explicarse por el hecho de que estas son menos frecuentes y variadas que las de los participantes con un nivel más alto de educación. Desafortunadamente, la encuesta estaba limitada por espacio y tiempo de aplicación, y no se pudo recopilar información sobre el tipo de actividades de ocio que suelen realizar los participantes. Finalmente, respecto de la edad, los participantes mayores de 60 años reportan una probabilidad mayor de cambiar las actividades de ocio y evitar la realización de actividades al aire libre cuando perciben que el aire de la ciudad está contaminado. A pesar de que este resultado indica cierto nivel de autoprotección en un grupo especialmente vulnerable, hay que destacar su adhesión relativamente baja al resto de conductas (especialmente, uso de mascarilla, mudarse y evitar la apertura de ventanas).

Respecto a los factores psicosociales, cabe destacar la percepción de riesgo como principal variable predictora. Los sujetos que percibieron como más graves los efectos de la contaminación del aire sobre la salud fueron quienes más reportaron un cambio en sus actividades de ocio y evitar la apertura de ventanas; también fueron quienes más evitaron realizar ejercicio al aire libre durante el último invierno. 
Asimismo, las personas que relacionan la contaminación del aire con efectos sobre la salud a corto plazo fueron quienes tuvieron mayor probabilidad de cambiar sus actividades de ocio.

Finalmente, los resultados muestran que los participantes que prestan mayor atención a la calidad del aire que se respira al salir de casa o del trabajo, suelen ser los que, en mayor medida, evitan realizar ejercicios al aire libre, abrir las ventanas y, en menor proporción, cambian sus actividades de ocio. De igual forma, los sujetos que evaluaron como muy mala la calidad del aire fueron quienes cambiaron sus actividades de ocio y pensaron en mudarse de casa. Estos hallazgos refuerzan estudios previos que indicaron una relación entre factores actitudinales (conciencia, atención prestada a la calidad del aire y percepción de riesgo) y una mayor adherencia a conductas de protección, especialmente aquellas tendientes a aminorar la exposición frente a la contaminación (Qian et al. 2016, Ma et al. 2018).

\section{CONCLUSIONES}

Esta investigación muestra que no es posible entender las estrategias individuales de protección frente a la contaminación urbana del aire sin atender el papel que desempeñan los factores sociodemográficos y actitudinales. Los resultados de este estudio muestran que las personas suelen adherirse en mayor medida a las conductas que tienen una mayor presencia en las campañas de sensibilización o bien un bajo coste percibido. A escala individual, el género, la edad, la educación, la atención y la percepción de riesgo a la salud son variables clave para entender la variación de las conductas de autoprotección frente a la mala calidad del aire. Ante el carácter voluntario y la ausencia de legislación y seguimiento institucional, los hallazgos reportados en este artículo pueden ayudar a la generación de programas de prevención más efectivos, dirigiendo los esfuerzos hacia los estratos poblacionales menos comprometidos con su autocuidado o incentivando, a través de medidas regulatorias, aquellas acciones que socialmente son peor percibidas. El cambio experimentado en la región con el uso de las mascarillas, residual antes de la pandemia del coronavirus, muestra que es posible transformar los hábitos poblacionales con una adecuada comunicación de riesgo y sin necesidad de excesiva fiscalización. Ante las dificultades de las ciudades del sur de Chile para transitar a un escenario de mayor sostenibilidad, el diseño de políticas de prevención debería fundamentarse en evidencia científica, con el fin de lograr una mayor implicación ciudadana en el control de la contaminación atmosférica.

\section{AGRADECIMIENTOS}

Los autores de este estudio agradecen a la Dra. Ana Barrera Herrera sus comentarios realizados a versiones previas de este documento. Este artículo es parte del proyecto Fondecyt Regular 1190412, y fue parcialmente financiado por la Agencia Nacional de Investigación y Desarrollo (ANID), Chile, y la Dirección de Investigación de la Universidad de la Frontera, a través de su Concurso de Ayuda a Grupos de Alta Productividad. Finalmente, los autores agradecen a las y los participantes que colaboraron voluntariamente en la encuesta.

\section{REFERENCIAS}

Álvarez B. y Boso À. (2018). Representaciones sociales de la contaminación del aire y las estufas de leña. Rev. Int. Contam. Ambie. 34 (3), 527-540. https://doi. org/10.20937/RICA.2018.34.03.14

Aragón F.M., Miranda J.J. y Oliva P. (2017). Particulate matter and labor supply: The role of caregiving and non-linearities. J. Environ. Econ. Manag. 86, 295-309. https://doi.org/10.1016/j.jeem.2017.02.008Get

Armijo G., Whitman C. y Roubelat L. (2013). Reacondicionamiento térmico en Temuco-Padre Las Casas, Chile: una ciudad declarada saturada por contaminación aérea. Memorias. XII Encontro Nacional de Conforto no Ambiente Construido ENCAC y VIII Encontro Latinoamericano de Conforto no Ambiente Construido ELACAC. Brasilia, septiembre.

Barría R., Calvo M. y Pino P. (2016). Contaminación intradomiciliaria por material particulado fino (MP2.5) en hogares de recién nacidos. Rev. Chil. Pediatr. 87 (5), 343-350. https://doi.org/10.1016/j. rchipe.2016.04.007

Bell M.L., Belanger K., Ebisu K., Gent J., Joo H., Koutrakis P. y Leaderer B. (2010). Prenatal exposure to fine particulate matter and birth weight: variations by particulate constituents and sources. Epidemiology 21 (6), 884-891. https://doi.org/10.1097/ EDE.0b013e3181f2f405

Boso À., Álvarez B., Oltra C., Garrido J., Muñoz C. y Hofflinger Á. (2020a). Out of sight, out of mind: participatory sensing for monitoring indoor air quality. Environ. Monit. Assess. 192, 104. https://doi. org/10.1007/s10661-019-8058-Z 
Boso À., Garrido J., Álvarez B., Oltra C., Hofflinger A. y Gálvez G. (2020b). Narratives of resistance to technological change: drawing lessons for urban energy transitions in Southern Chile. Energy Research and Social Sciences 65, 101473. https://doi.org/10.1016/j. erss.2020.101473

Brook R.D., Rajagopalan S., Pope 3rd C.A., Brook J.R., Bhatnagar A., Diez-Roux A.V., et al. (2010). Particulate matter air pollution and cardiovascular disease: an update to the scientific statement from the American heart association. Circulation 121 (21), 2331-2378. https://doi.org/10.1161/cir.0b013e3181dbece1

Burnett R., Hong C., Szyszkowicz M., Fann N., Hubbel B., Pope 3rd C.A., et al. (2018). Global estimates of mortality associated with long-term exposure to outdoor fine particulate matter. Proc. Natl. Acad. Sci. USA 115 (38), 9592- 9597. https://doi.org/10.1073/ pnas. 1803222115

Cai D. y He Y. (2016). Daily lifestyles in the fog and haze weather. J. Thorac. Dis. 8 (1), 75-77. https://doi. org/10.3978/j.issn.2072-1439.2016.01.35

Casas L., Cox B., Bauwelinck M., Nemery B., Deboosere P. y Nawrot T.S. (2017). Does air pollution trigger suicide? A case-crossover analysis of suicide deaths over the life span. Eur. J. Epidemiol. 32 (11), 973-981. https://doi.org/10.1007/s10654-017-0273-8

Chang T., Graff Zivin J., Gross T. y Neidell M. (2016). Particulate pollution and the productivity of pear packers. Am. Econ. J. Econ. Polic. 8 (3), 141-169. https:// doi.org/10.1257/pol.20150085

Chung Y., Alves C. y Rappenglück B. (2019). Whether to wear a pollution filter. Air Qual. Atmos. Hlth. 12 (7), 765-766. https://doi.org/10.1007/s11869-019-00713-9

Corona J. (2016). Apuntes sobre métodos de investigación. Medisur 14 (1), 81-83.

Cortés A. y Ridley I. (2013). Efectos de la combustión a leña en la calidad del aire intradomiciliario: la ciudad de Temuco como caso de estudio. Revista INVI 28 (78), 257-271. https://doi.org/10.4067/S071883582013000200008

Currie J., Hanushek E., Kahn E.M., Neidell M. y Rivkin S. (2009). Does pollution increase school absences? Rev. Econ. Stat. 91, 682-694. https://doi.org/10.1162/ rest.91.4.682

Cutter S.C. (1981). Community concern for pollution: social and environmental influences. Environ. Behav. 13 (1), 105-124. https://doi.org/10.1177/0013916581131006

D’Antoni D., Smith L., Auyeung V. y Weinman J. (2017). Psychosocial and demographic predictors of adherence and non-adherence to health advice accompanying air quality warning systems: a systematic review. Environ. Health-Glob. 16, 100. https://doi.org/10.1186/s12940017-0307-4
Deguen S., Ségala C., Pédrono G. y Mesbah M. (2012). A new air quality perception scale for global assessment of air pollution health effects. Risk Anal. 32 (12), 2043-2054. https://doi.org/10.1111/j.15396924.2012.01862.x

Díaz-Narváez V. y Calzadilla A. (2016). Artículos científicos, tipos de investigación y productividad científica en las Ciencias de la Salud. Ciencias de la Salud 14 (1), 115-121. https://doi.org/10.12804/ revsalud14.01.2016.10

Díaz-Robles L.A., Ortega J.C., Fu J.S., Reed G., Chow J., Watson J. y Moncada J. (2018). A hybrid ARIMA and artificial neural networks model to forecast particulate matter in urban areas: the case of Temuco, Chile. Atmos. Environ. 42 (35), 8331-8340. https:// doi.org/10.1016/j.atmosenv.2008.07.020

Dominici F., Peng R., Bell M., Pham L., McDermott A., Zeger S. y Samet J. (2006). Fine particulate air pollution and hospital admission for cardiovascular and respiratory diseases. J. Am. Med. Assoc. 295 (19), 1127-1134. https://doi.org/10.1001/jama.295.10.1127

Evans G. y Jacobs S. (1981). Air pollution and human behavior. J. Soc. Issues 37 (1), 95-125. https://doi. org/10.1111/j.1540-4560.1981.tb01059.x

Finucane M.L., Alhakami A., Slovic P.y Johnson S.M. (2000). The affect heuristic in judgments of risks and benefits. J. Behav. Decis. Mak. 13(1), 1-17. https://doi.org/10.1002/ (SICI)1099-0771(200001/03)13:13.0.CO;2-S

Fischer G.W., Morgan M.G., Fischhoff B., Nair I. y Lave L.B. (1991). What risks are people concerned about. Risk Anal. 11 (2), 303-314. https://doi. org/10.1111/j.1539-6924.1991.tb00606.x

Flynn J., Slovic P. y Mertz C.K. (1994). Gender, race, and perception of environmental health risks. Risk Anal. 14 (6), 1101-1108. https://doi. org/10.1111/j.1539-6924.1994.tb00082.x

Gurajala S. y Matthews J. (2018). Twitter data analysis to understand societal response to air quality. Memorias. 9th International on Social Media and Society ' 18. ACM, Nueva York, julio, 82-90.

Guxens M. y Sunyer J. (2012). A review of epidemiological studies on neuropsychological effects of air pollution. Swiss Med. Wkly. 141 (1), w13322. https://doi. org/10.4414/smw.2012.13322

Henry G. y Gordon C. (2003). Driving less for better air: Impacts of a public information campaign. J. Policy Anal. Manag. 22 (1), 45-63. https://doi.org/10.1002/ pam. 10095

Hernández R., Fernández C. y Baptista P. (2014). Metodología de la investigación. 6a ed. McGraw-Hill, Ciudad de México, 632 pp.

Hosmer D. y Lemeshow S. (1989). Applied logistic regression. 2a ed. John Wiley \& Sons, Nueva York, 307 pp. 
Hu L., Zhu L., Xu Y., Lyu J., Imm K. y Yang L. (2017). Relationship between air quality and outdoor exercise behavior in China: A novel mobile-based study. Int. J. Behav. Med. 24 (4), 520-527. https://doi.org/10.1007/ s12529-017-9647-2

INE (2017). Resultados preliminares Censo 2017. Instituto Nacional de Estadísticas [en línea]. http://www. censo2017.cl/ 13/03/2021

IQair (2019). 2018 world air quality report region \& city PM2.5 ranking [en línea]. https://www.iqair.com/ world-most-polluted-cities 13/03/2021

Jacquemin B., Sunyer J., Forsberg B., Götschi T., BayerOglesby L., Ackermann-Liebrich U., et al. (2007). Annoyance due to air pollution in Europe. Int. J. Epidemiol. 36 (4), 809-820. https://doi.org/10.1093/ije/dym042

Johnson B.B. (2002). Gender and race in beliefs about outdoor air pollution. Risk Anal. 22 (4), 725-738. https://doi.org/10.1111/0272-4332.00064

Johnson B.B. (2012). Experience with urban air pollution in Paterson, New Jersey and Implications for air pollution communication. Risk Anal. 32 (1), 39-53. https:// doi.org/10.1111/j.1539-6924.2011.01669.x

Kan H., London S., Chen G., Zhang Y., Song G., Zhao N., Jiang, L. y Chen B. (2008). Season, sex, age, and education as modifiers of the effects of outdoor air pollution on daily mortality in Shanghai, China: The Public Health and Air Pollution in Asia (PAPA) study. Environ. Health Perspect. 116 (9), 1183-1188. https:// doi.org/10.1289/ehp.10851

Kim C. y Hu S. (1998). Regional deposition of inhaled particles in human lungs: comparison between men and women. J. Appl. Physiol. 84 (6), 1834-1844. https:// doi.org/10.1152/jappl.1998.84.6.1834

Kim Y., Ng C.F.S., Chung Y., Kim H., Honda Y., Guo Y.L., et al. (2018). Air pollution and suicide in 10 cities in Northeast Asia: a time-stratified case-crossover analysis. Environ. Health Perspect. 126 (3), 037002. https://doi.org/10.1289/EHP2223.

Langer S., Ramalho O., Le Ponner E., Derbez M., Kirchner S. y Mandin C. (2017). Perceived indoor air quality and its relationship to air pollutants in French dwellings. Indoor Air 27 (6), 1168-1176. https://doi.org/10.1111/ ina. 12393

Laumbach R., Meng Q. y Kipen H. (2015). What can individuals do to reduce personal health risks from air pollution? J. Thorac. Dis. 7 (1), 96-107. https://doi. org/10.3978/j.issn.2072-1439.2014.12.21

Lee H., Myung W., Kim S.E., Kim D.K. y Kim H. (2018). Ambient air pollution and completed suicide in 26 South Korean cities: Effect modification by demographic and socioeconomic factors. Sci. Total Environ. 639, 944-951. https://doi.org/10.1016/j.scitotenv.2018.05.210
Liao X., Tu H., Maddock J., Fan S., Lan G., Wu Y., et al. (2015). Residents' perception of air quality, pollution sources, and air pollution control in Nanchang, China. Atmos. Pollut. Res. 6 (5), 835-841. https://doi. org/10.5094/apr.2015.092

Lin T., Li L. y Bautista J. (2016). Examining how communication and knowledge relate to singaporean youths' perceived risk of haze and intentions to take preventive behaviors. Health Commun. 32 (6), 749-758. https:// doi.org/10.1080/10410236.2016.1172288

Lindell M. y Perry R. (1992). Behavioral foundations of community emergency planning. Taylor and Francis. Washington DC, $630 \mathrm{pp}$.

Lissåker C., Talbott E., Kan H. y Xu X. (2014). Status and determinants of individual actions to reduce health impacts of air pollution in U.S. adults. Arch. Environ. Occup. Health 71 (1), 44-48. https://doi.org/10.1080/ 19338244.2014.988673

Liu H. y Salvo A. (2018). Severe air pollution and child absences when schools and parents respond. J. Environ. Econ. Manage. 92, 300-330. https://doi.org/10.1016/j. jeem.2018.10.003

Long J. (1997). Regression models for categorical and limited dependent variables. Bloomington, SAGE Publications. Londres, $297 \mathrm{pp}$.

Ma Y., Yuan B., Fan S., Luo Y. y Wen X. (2018). Association between air quality and Sedentary time in 3270 Chinese adults: Application of a novel technology for posture determination. J. Clin. Med. 7 (9), 257. https:// doi.org/10.3390/jcm7090257

Medina-Ramón M., Zanobetti A. y Schwartz J. (2006). The effect of ozone and $\mathrm{PM}_{10}$ on hospital admissions for pneumonia and chronic obstructive pulmonary disease: a national multicity study. Am. J. Epidemiol. 163 (6), 579-588. https://doi.org/10.1093/aje/kwj078

Meteored (2019). Datos históricos en Temuco [en línea]. https://www.meteored.cl/tiempo-en_TemucoAmerica+Sur-Chile-Araucania-SCTC-sactual-18267. html

ME (2015). Medición del consumo nacional de leña y otros combustibles sólidos derivados de la madera. Informe. Ministerio de Energía. Santiago, Chile, 302 pp.

Minsal (2015). Guía de recomendaciones de actividad física con alerta ambiental $\left(\mathrm{MP}_{2.5}\right)$. Guía. Ministerio de Salud. Santiago, Chile, 2 pp.

MMA (2014). Planes de descontaminación atmosférica: estrategia 2014-2018. Reporte. Ministerio del Medio Ambiente. Santiago, Chile, $18 \mathrm{pp}$.

MMA (2015). Decreto 8 Id 1084085. Establece plan de descontaminación atmosférica por $\mathrm{MP}_{2.5}$, para las comunas de Temuco y Padre las Casas. Actualización del plan de descontaminación por $\mathrm{MP}_{10}$, para las mismas comunas. Ministerio del Medio Ambiente, 
Diario Oficial de la República de Chile, $\mathrm{n}^{\circ}$ 41.309. 17 de noviembre.

MMA (2018). Reporte mensual evolución de episodios críticos para $\mathrm{MP}_{2.5}$. Reporte mensual septiembre. Ministerio del Medio Ambiente, Santiago, Chile, 8 pp.

MMA (2019a). Aire Chile. Recomendaciones. Ministerio del Medio Ambiente [en línea]. http://airechile.mma. gob.cl/recomendaciones 08/07/2020

MMA (2019b). Temuco y Padre Las Casas, calidad del aire. Ministerio del Medio Ambiente [en línea]. http:// airechile.mma.gob.cl/ 13/03/2021

Molina E., Brown L., Prieto V., Bonet M. y Cuéllar L. (2001). Crisis de asma y enfermedades respiratorias agudas: Contaminantes atmosféricos y variables meteorológicas en Centro Habana. Rev. Cubana Med. Gen. Integr. 17 (1), 10-20.

Neidell M. (2006). Public information and avoidance behavior: Do people respond to smog alerts? Reporte técnico. Center for Integrating Statistical and Environmental Science, The University of Chicago, $39 \mathrm{pp}$.

Noonan D.S. (2011). Smoggy with a chance of altruism: Using air quality forecasts to drive behavioral change. Documento de trabajo. National Research Initiative and American Enterprise Institute, Georgia, EUA, 17 pp.

Oliveira M.S., Leon A.P., Mattos I.E. y Koifman S. (2011). Differential susceptibility according to gender in the association between air pollution and mortality from respiratory diseases. Cad. Saude Publica. 27 (9), 1827-1836. https://doi.org/10.1590/s0102$311 \times 2011000900016$

Oltra C. y Sala R. (2016). Perception of risk from air pollution and reported behaviors: a cross-sectional survey study in four cities. J. Risk Res. 21 (7), 869-884. https:// doi.org/10.1080/13669877.2016.1264446

Oltra C., Sala R., Boso À. y Asensio S.L. (2017). Public engagement on urban air pollution: an exploratory study of two interventions. Environ. Monit. Assess. 189 (6), 296. https://doi.org/10.1007/s10661-0176011-6

OMS (2019). Calidad del aire y salud. Organización Mundial de la Salud [en línea]. https://www.who.int/ es/news-room/fact-sheets/detail/ambient-(outdoor)air-quality-and-health 07/08/2020.

Peng R.D., Bell M.L., Geyh A., McDermott A., Zeger S., Samet J. y Dominici F. (2009). Emergency admissions for cardiovascular and respiratory diseases and the chemical composition of fine particle air pollution. Environ. Health Perspect. 117 (6), 957-963. https:// doi.org/10.1289/ehp.0800185

Qian X., Xu G., Li L., Shen Y., He T., Liang Y., et al. (2016). Knowledge and perceptions of air pollution in Ningbo, China. BMC Public Health 16 (1), 1138. https://doi.org/10.1186/s12889-016-3788-0
Qin C., Xu J., Wong-Parodi G. y Xue L. (2018). Change in public concern and responsive behaviors toward air pollution under the dome. Risk Anal. 40 (10), 19832001. https://doi.org/10.1111/risa. 13177

Saksena S. (2011). Public perceptions of urban air pollution risks. Risk, Hazards and Crisis in Public Policy 2 (1), 1-19. https://doi.org/10.2202/1944-4079.1075

Sala R., Oltra C. y Gonçalves L. (2014). Percepción pública de la contaminación atmosférica urbana: un análisis exploratorio. Informe técnico. Centro de Investigaciones Energéticas, Medioambientales y Tecnológicas. Madrid, España, 120 pp.

Sala R., López-Asensio S., Moya E. y Oltra C. (2017). Percepción pública de la contaminación atmosférica urbana en Pamplona. Informe técnico. Centro de Investigaciones Energéticas, Medioambientales y Tecnológicas. Madrid, España, 120 pp.

Schueftan A. y González A. (2015). Proposals to enhance thermal efficiency programs and air pollution control in south-central Chile. Energ. Policy. 79 (2015), 48-57. https://doi.org/10.1016/j.enpol.2015.01.008

Semenza J., Wilson D., Parra J., Bontempo B., Hart M., Sailor D. y George L. (2008). Public perception and behavior change in relationship to hot weather and air pollution. Environ. Res. 107 (3), 401-411. https://doi. org/10.1016/j.envres.2008.03.005

Sun C., Kahn M. y Zheng S. (2017). Self-protection investment exacerbates air pollution exposure inequality in urban China. Ecol. Econ. 131, 468-474. https://doi. org/10.1016/j.ecolecon.2016.06.030

Unosson J., Blomberg A., Sandström T., Muala A., Boman C., Nyström R., et al. (2013). Exposure to wood smoke increases arterial stiffness and decreases heart rate variability in humans. Part Fibre. Toxicol. 10 (1), 20. https://doi.org/10.1186/1743-8977-10-20

Weather Spark. (2019). El clima promedio en Temuco Chile [en línea]. https://es.weatherspark.com/y/25123/ Clima-promedio-en-Temuco-Chile-durante-todo-ela\%C3\%B1o 08/07/2020

Wells E.M., Dearborn D.G. y Jackson L. (2012). Activity change in response to bad air quality. National Health and Nutrition Examination Survey, 2007-2010. PLoS ONE 7 (11), e50526. https://doi.org/10.1371/journal. pone. 0050526

Yu H., Yu M., Gordon S. y Zhang R. (2017). The association between ambient fine particulate air pollution and physical activity: a cohort study of university students living in Beijing. Int. J. Behav. Nutr. Phys. Act. 14 (1), 136. https://doi.org/10.1186/ s12966-017-0592-x

Yu H., Wu Y., Gordon S., Cheng J., Chen P., Wang Y. y Yu M. (2019). Objectively measured the impact of air pollution on physical activity and sedentary behavior in 
freshmen student in Beijing, China. Research Square. https://doi.org/10.21203/rs.2.12847/v1

Zehaie F. (2009). The timing and strategic role of self-protection. Environmental and Resource Economics 44 (3), 337- 350. https://doi.org/10.1007/s10640-009-9289-1

Zhang J. y Mu Q. (2018). Air pollution and defensive expenditures: Evidence from particulate-filtering facemasks. J. Environ. Econ. Manag. 92, 517-536. https://doi.org/10.1016/j.jeem.2017.07.006

Zhou S., Lukula S., Chiossone C., Nims R., Suchmann D. y Ijaz M. (2018). Assessment of a respiratory face mask for capturing air pollutants and pathogens including human influenza and rhinoviruses. J. Thorac. Dis. 10 (3), 2059-2069. https://doi.org/10.21037/jtd.2018.03.103 